\title{
Designing of Hybrid form of Benzothiazole-quinazoline as GABA-A Inhibitor with Anticonvulsant Profile: An in-silico Approach
}

\author{
Ajeet ${ }^{*}$, Arvind Kumar \\ Department of Pharmaceutical Chemistry and Drug Design, S. D. College of Pharmacy and Vocational Studies, Muzaffarnagar, India \\ *Corresponding author: ajeet_pharma111@rediffmail.com
}

Received October 16, 2013; Revised December 08, 2013; Accepted December 24, 2013

\begin{abstract}
Epilepsy is a common but serious brain disorder. It is universal, with no age, sex, geographical, social class or racial boundaries. One of the most important mechanisms for handling it is the inhibition of the GABA-A receptor. In same context while studying the treatment of epilepsy we found the significant effects of the derivatives of the benzothiazole and quinazolines, this promotes us to develop a hybrid form of these two moieties by the means of in-silico resources with antiepileptic/anticonvulsant effects. Molecular docking approaches are routinely used in modern drug design to help understand drug-receptor interaction. This study has been performed with the help of Chemdraw Ultra 7.0, AutoDock Vina (Python Prescription 0.8), and PaDEL software. Results revealed that ligandprotein interaction affinity of all designed 10 hybrid molecules ranges from $-6.8 \mathrm{Kcal} / \mathrm{mol}$ to $-6.2 \mathrm{Kcal} / \mathrm{mol}$ which is approximately double as compared to pre-existing GABA-A inhibitor i.e. $\gamma$-aminobutyric acid (CID: 119, ligandprotein interaction affinity is $-3.2 \mathrm{Kcal} / \mathrm{mol})$.
\end{abstract}

Keywords: Benzothiazole-quinazolines, docking, GABA-A inhibitors, $\gamma$-aminobutyric acid, protein-ligand interaction affinity

Cite This Article: Ajeet, and Arvind Kumar, "Designing of Hybrid form of Benzothiazole-quinazoline as GABA-A Inhibitor with Anticonvulsant Profile: An in-silico Approach.” American Journal of Pharmacological Sciences 1, no. 6 (2013): 116-120. doi: 10.12691/ajps-1-6-2.

\section{Introduction}

Epilepsy is a common but serious brain disorder. It is universal, with no age, sex, geographical, social class or racial boundaries. Epilepsy imposes a large economic burden on health care systems of countries. There is also a hidden burden associated with stigma and discrimination against the patient and even his/her family in the community, workplace, and school and home. Many patients with epilepsy suffer severe emotional distress, behavioral disorders and extreme social isolation [1]. Epilepsy is usually defined as a tendency to recurrent seizures. The word "epilepsy" is derived from Latin and Greek words for "seizure” or "to seize upon”. This implies that epilepsy is an ancient disorder; indeed, in all civilizations it can be traced as far back as medical records exist. In fact, epilepsy is a disorder that can occur in all mammalian species, probably more frequently as brains have become more complex. Epilepsy is also remarkably uniformly distributed around the world. There are no racial, geographical or social class boundaries. It occurs in both sexes, at all ages, especially in childhood, adolescence and increasingly in ageing populations [2]. The term seizure refers to a transient alteration of behavior due to the disordered, synchronous, and rhythmic firing of population of the brain neurons [3]. The meaning of the term seizure needs to be carefully distinguished from that of epilepsy. Epilepsy describes a condition in which a person has recurrent seizures due to a chronic, underlying process. This definition implies that a person with a single seizure, or recurrent seizures due to correctable or avoidable circumstances, does not necessarily have epilepsy. Epilepsy refers to a clinical phenomenon rather than a single disease entity, since there are many forms and causes of epilepsy. However, among the many causes of epilepsy there are various epilepsy syndromes in which the clinical and pathologic characteristics are distinctive and suggest a specific underlying etiology [4].

The GABA-A receptor is an ionotropic receptor and ligand-gated ion channel. Its endogenous ligand is $\gamma$ aminobutyric acid (GABA (CID: 119)), the major inhibitory neurotransmitter in the central nervous system. Upon activation, the GABA-A receptor selectively conducts $\mathrm{Cl}^{-}$through its pore, resulting in hyperpolarization of the neuron. This causes an inhibitory effect on neurotransmission by diminishing the chance of a successful action potential occurring [5].

Candidates from benzothiazoles for designing novel anticonvulsant agents emerge with promising effects. The endocyclic nitrogen and sulphur functions present in the heterocyclic nucleus have been shown to be critical for anticonvulsant activity. [6,7] Harish Rajak et al confirmed by the preliminary pharmacological animal models that semicarbazones containing quinazoline ring emerges with a novel anticonvulsant series [8]. 
On studying the above mentioned literature about the anticonvulsant behavior of benzothiazoles and quinazolines promoted us to theoretically develop a hybrid of these two moieties by the means of in-silico resources.

Rational drug design helps to facilitate and speedup the drug designing process, which involves variety of methods to identify novel compound, out of them one method is the docking of the drug molecule with the receptor. The therapeutic action of the clinical drug will be effective when the biochemical pathway of the enzyme can be exploited [9,10,11,12].

Docking procedures allows virtually screening a database of compounds and predict the strongest binder based on various scoring functions. It gives way in which two molecules such as drugs and an enzyme receptor fit together and dock to each other well $[9,10,11,12]$.

Molecular docking techniques are used in modern drug design to help understand drug-receptor interaction. It has been shown in the literature that these computational procedures can strongly support and help the design of new, more potent drugs by revealing the mechanism of drug-receptor interaction [9,10,11,12].

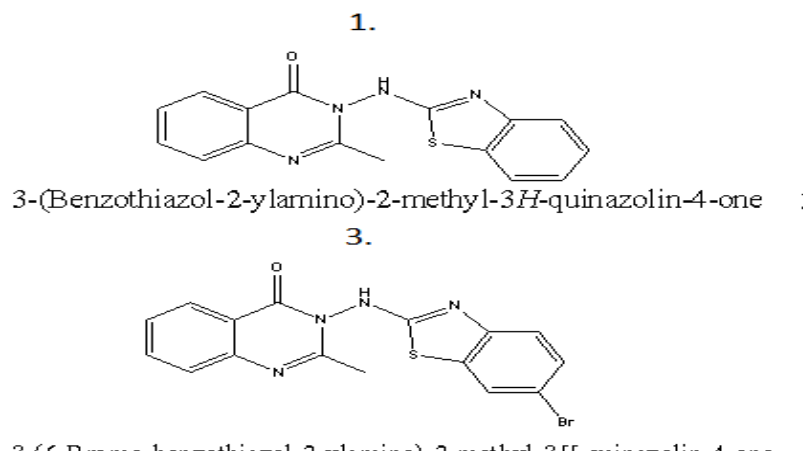

3-(6-B romo-benzothiazol-2-ylamino)-2-methyl-3F-quinazolin-4-one 5.

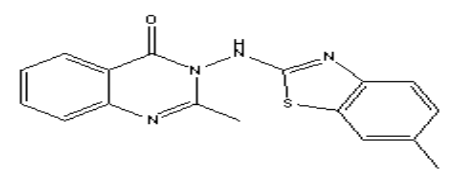

2-Methyl-3-(6-methyl-benzothiazol-2-ylamino)-3H-quinazolin-4-one 7.

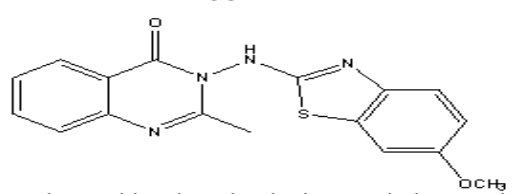

3-(6-Methoxy-benzothiazol-2-ylamino)-2-methyl-3H-quinazolin-4-one 9.

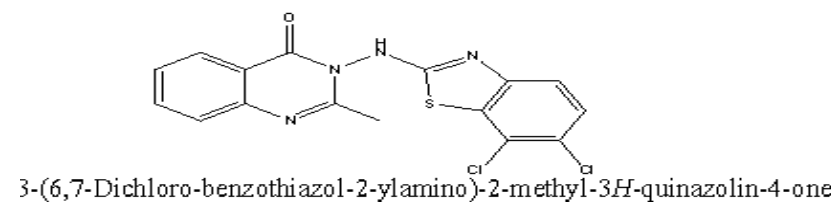

\section{Materials and Method}

\subsection{Database and Tools}

For carrying out the study, National Center for Biotechnology Information's (NCBI.) website and Protein Data Bank's (PDB) website were used as biological and receptor sources. For designing and optimizing the geometry of the derivatives, Chemdraw Ultra 7.0 [13] was used. For docking studies of derivatives, AutoDock Vina $[10,11,12]$ molecular docking software has been employed and for descriptor calculations PaDEL software has been used [14].

\subsection{Molecule Designing and Optimization}

The chemical structures of the hybrid derivatives Figure 1 were drawn using ChemDraw Ultra 7.0 and energy minimization of derivatives was achieved with Chem3D Pro of ChemOffice suit for taking energy of each molecule up to its lowest energy state (highest stability). 3D structure of gamma aminobutyric acid (CID: 119) was retrieved from PubChem compound database at NCBI.

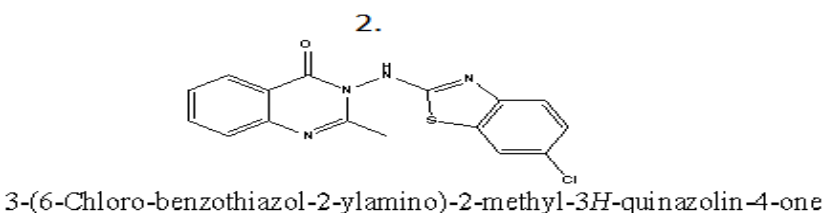

4.

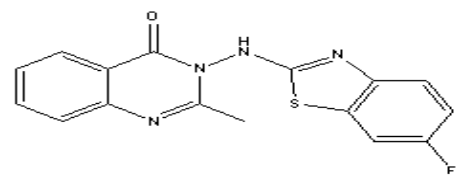

3-(6-Fluoro-benzo thiazol-2-ylamino)-2-methyl-3H-quinazolin-4-one

6.

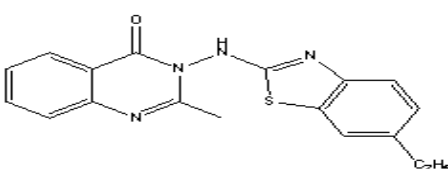

3-(6-Ethyl-benzothiazol-2-ylamino)-2-methyl-3H-quinazolin-4-one

8.

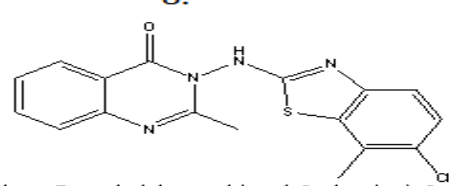

3-(6-Chloro-7-miethyl-benzotfiazol-2-ylamino )-2-methyl-3 4 -quinazolin-4-one 10.

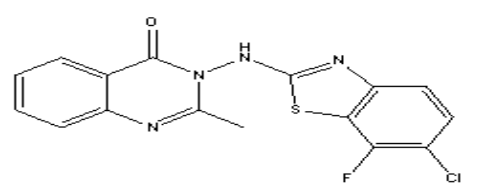

3-(6-Chloro-7-fluor o-berz othiæa ol-2-yl amino) -2 -methyl-3H-quinazolin-4-one

Figure 1. Chemical structures of the hybrid derivatives

\subsection{Docking Studies}

The docking analysis of hybrid derivatives with GABA-A was carried out by AutoDock Vina. The incorporation of various algorithms makes it a very good tool, as docking search algorithm is based on evolutionary algorithm. It is an iterative optimization technique inspired by Darwinian evolution theory. Evolutionary algorithm consists of population of individuals, which is exposed to random variation by means of variation operators, like mutation and recombination.

The designed hybrid benzothiazole-quinazoline derivatives were docked into binding site of GABA-A receptor. For this study, X-ray crystal structure of GABAA receptor was retrieved from protein data bank.

\section{Results and Discussion}




\subsection{Docking Results}

\subsubsection{Binding site Analysis}

The experimental analysis of binding site shows that PHE 334, TRP 266 and SER 137 could be the catalytic site residue present in the structure of GABA-A receptor.

\subsubsection{Docking Studies of Hybrid Benzothiazole- Quinazoline Derivatives with GABA-A Receptor}

The protein-ligand interaction affinity of hybrid benzothiazole-quinazoline inhibitors were given by
AutoDock Vina for best pose of novel inhibitors. The best pose ligand-protein interaction affinity of 10 molecules was found to be as $-6.2 \mathrm{Kcal} / \mathrm{mol},-6.5 \mathrm{Kcal} / \mathrm{mol},-6.3$ $\mathrm{Kcal} / \mathrm{mol},-6.4 \mathrm{Kcal} / \mathrm{mol},-6.4 \mathrm{Kcal} / \mathrm{mol},-6.8 \mathrm{Kcal} / \mathrm{mol}$, $6.4 \mathrm{Kcal} / \mathrm{mol},-6.8 \mathrm{Kcal} / \mathrm{mol},-6.7 \mathrm{Kcal} / \mathrm{mol}$ and -6.5 $\mathrm{Kcal} / \mathrm{mol}$ respectively. Here, negative values for interaction energy would reflect the positive docking approach. Number of hydrogen bonds and other binding details are given in Table 1 and docking images are given in Figure 2.

1.
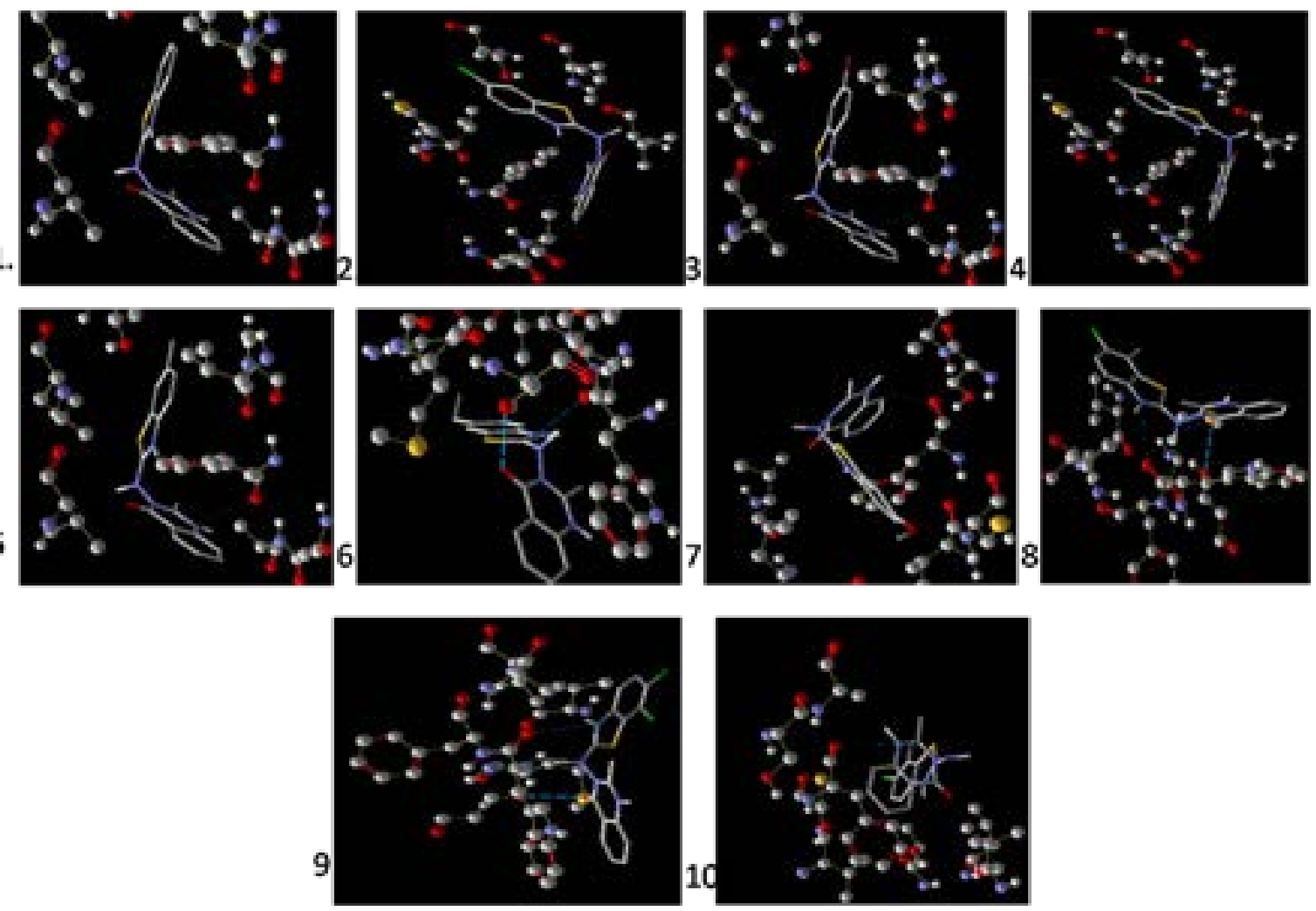

Figure 2. Docked photographs of hybrid benzothiazole-quinazoline derivatives with GABA-A receptor

Table 1. Docking results of hybrid benzothiazole-quinazoline derivatives and $\gamma$-aminobutyric acid (CID: 119)

\begin{tabular}{|c|c|c|c|c|c|c|c|c|c|c|}
\hline \multirow[t]{2}{*}{ Ligand } & \multirow[t]{2}{*}{ Receptor } & \multirow[t]{2}{*}{$\begin{array}{c}\text { Affinity } \\
\text { Kcal/mol } \\
\end{array}$} & \multirow[t]{2}{*}{ H-bonds } & \multicolumn{3}{|c|}{ H- Binding Ligand } & \multicolumn{4}{|c|}{ H- Binding Receptor } \\
\hline & & & & Elem. & At. ID. & Type & Res. & Elem. & At.ID. & Type \\
\hline 1 & \multirow{18}{*}{ GABA(A) alpha-1 } & -6.2 & 1 & $\mathrm{~N}$ & 06 & Donor & PHE & $\mathrm{O}$ & 334 & Acceptor \\
\hline 2 & & -6.5 & 1 & $\mathrm{~N}$ & 06 & Donor & PHE & $\mathrm{O}$ & 334 & Acceptor \\
\hline 3 & & -6.3 & 1 & $\mathrm{~N}$ & 06 & Donor & PHE & $\mathrm{O}$ & 334 & Acceptor \\
\hline 4 & & -6.4 & 1 & $\mathrm{~N}$ & 06 & Donor & PHE & $\mathrm{O}$ & 334 & Acceptor \\
\hline 5 & & -6.4 & 1 & $\mathrm{~N}$ & 06 & Donor & PHE & $\mathrm{O}$ & 334 & Acceptor \\
\hline \multirow{2}{*}{6} & & \multirow{2}{*}{-6.8} & \multirow{2}{*}{2} & $\mathrm{~N}$ & 20 & Acceptor & TRP & $\mathrm{O}$ & 266 & Acceptor \\
\hline & & & & $\mathrm{N}$ & 20 & Donor & SER & $\mathrm{O}$ & 137 & Both \\
\hline 7 & & -6.4 & 1 & $\mathrm{~N}$ & 04 & Donor & PHE & $\mathrm{O}$ & 334 & Acceptor \\
\hline \multirow{2}{*}{8} & & \multirow{2}{*}{-6.8} & \multirow[b]{2}{*}{2} & $\mathrm{~N}$ & 20 & Donor & TRP & $\mathrm{O}$ & 266 & Acceptor \\
\hline & & & & $\mathrm{O}$ & 14 & Acceptor & SER & $\mathrm{O}$ & 137 & Both \\
\hline \multirow{2}{*}{9} & & \multirow{2}{*}{-6.7} & \multirow{2}{*}{2} & $\mathrm{~N}$ & 20 & Donor & TRP & $\mathrm{O}$ & 266 & Acceptor \\
\hline & & & & $\mathrm{O}$ & 14 & Acceptor & SER & $\mathrm{O}$ & 137 & Both \\
\hline 10 & & -6.5 & 1 & $\mathrm{~N}$ & 04 & Donor & PHE & $\mathrm{O}$ & 334 & Acceptor \\
\hline \multirow{5}{*}{ CID_119 } & & \multirow{5}{*}{-3.2} & \multirow{5}{*}{5} & $\mathrm{~N}$ & 07 & Donor & LEU & $\mathrm{O}$ & 127 & Acceptor \\
\hline & & & & $\mathrm{O}$ & 04 & Both & SER & $\mathrm{O}$ & 135 & Acceptor \\
\hline & & & & $\mathrm{O}$ & 04 & Both & ALA & $\mathrm{O}$ & 216 & Acceptor \\
\hline & & & & $\mathrm{O}$ & 04 & Both & THR & $\mathrm{O}$ & 240 & Both \\
\hline & & & & $\mathrm{O}$ & 03 & Acceptor & THR & $\mathrm{O}$ & 240 & Both \\
\hline
\end{tabular}




\subsection{Comparison of Docking Results with Pre- existing GABA-A Inhibitor $\gamma$-aminobutyric acid (GABA CID: 119)}

On docking studies and docking analysis of $\gamma$ aminobutyric acid with the GABA-A, interacting residues (amino acids) are found as THR 311, ALA 308, LEU 296, SER 299, ALA 312, TRP 315 and SER 297 Figure 3.

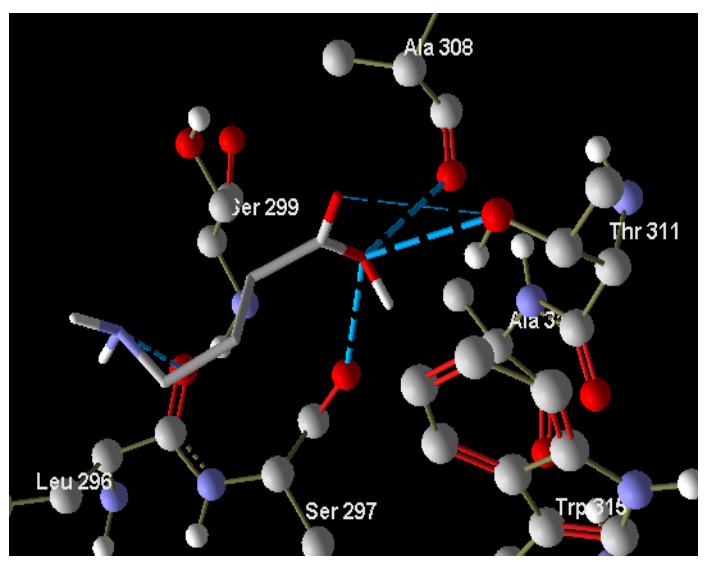

Figure 3. Docking photograph of $\gamma$-aminobutyric acid with the GABA-A showing interacting aminoacids

On docking analysis, the docked poses of all hybrid benzothiazole-quinazoline derivatives do not superimposes the $\gamma$-aminobutyric acid, a pre-existing GABA-A inhibitor which can be clearly seen in Figure 4, but the docking analysis shows that it nicely docked with protein rather in the catalytic domain for the $\gamma$-aminobutyric acid, it means that it binds in the allosteric site of the protein and there is possibilities of making it with anticonvulsant profile.

\subsection{Descriptor Studies}

All the 10 designed hybrid benzothiazole-quinazoline derivatives have been gone through some descriptor calculation, which are listed in Table 2.

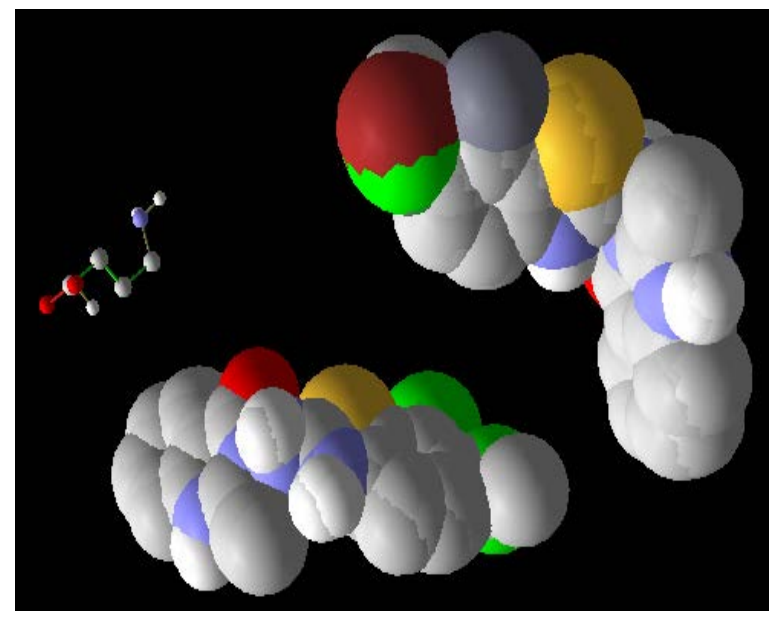

Figure 4. Non-superimposed docking poses of hybrid benzothiazolequinazoline derivatives (showing with spacefill model) with the preexisting ligand $\gamma$-aminobutyric acid (showing with ball and stick model)

Table 2. Descriptor studies of the hybrid benzothiazole-quinazoline derivatives

\begin{tabular}{|c|c|c|c|c|c|c|c|}
\hline S. No. & ALogP & XLogP & Lipinski Failures & Lipo-affinity Index & Eccentric connectivity index & $\begin{array}{c}\text { McGowan } \\
\text { Volume }\end{array}$ & Molecular weight \\
\hline $\mathbf{1}$ & 1.313 & 1.429 & 0 & -1.21666 & 426 & 1.905 & 295.9793 \\
\hline $\mathbf{2}$ & 1.6817 & 2.006 & 0 & -1.18291 & 476 & 2.0489 & 330.9481 \\
\hline $\mathbf{3}$ & 1.7657 & 2.182 & 0 & -1.28653 & 476 & 2.1015 & 374.8976 \\
\hline $\mathbf{4}$ & 1.2228 & 1.545 & 0 & 0.654387 & 476 & 1.9442 & 314.9777 \\
\hline $\mathbf{5}$ & 1.7594 & 1.821 & 0 & -1.25471 & 476 & 2.0029 & 307.9793 \\
\hline $\mathbf{6}$ & 1.4243 & 2.285 & 0 & -1.30539 & 530 & 2.1008 & 319.9793 \\
\hline $\mathbf{7}$ & 0.8144 & 1.669 & 0 & -1.26816 & 530 & 2.0616 & 323.9742 \\
\hline $\mathbf{8}$ & 2.1281 & 2.187 & 0 & -1.30537 & 497 & 2.1468 & 342.9481 \\
\hline $\mathbf{9}$ & 2.0504 & 2.583 & 0 & -1.19454 & 497 & 2.1928 & 365.917 \\
\hline $\mathbf{1 0}$ & 1.5915 & 2.122 & 0 & 0.739636 & 497 & 2.0881 & 349.9465 \\
\hline
\end{tabular}

\section{Conclusion}

Docking study of hybrid benzothiazole-quinazoline derivatives proved them potential GABA-A inhibitors. Although a systemic biochemical study is necessary to confirm the findings. When hybrid form of benzothiazolequinazoline derivatives were docked with GABA-A then all the 10 designed molecules were found with the higher affinity values (more than double affinity value) as compared to the $\gamma$ aminobutyric acid, so it could be concluded that these hybrid derivatives may be proved the better inhibitors of GABA-A for the anticonvulsant activity. On comparing the chemical structure of hybrid benzothiazole-quinazoline derivatives with $\gamma$-aminobutyric acid, a pre-existing GABA-A inhibitor; there is no structural similarity found except a amine group so it is concluded that this hybrid system may proved a novel class of this category.

\section{References}

[1] Satischandra P., Gururaj G., Mohammed Q. D., Senanayake N., Silpakit O., Dekker P.A., "Epilepsy: A manual for physicians". World Health Organization, New Delhi, 1-15, 2005.

[2] Reynolds E. H., "Epiilepsy in the world: Launch of the second phase of the ILAE/IBE/WHO global campaign against epilepsy". Epilepsia, 43 (supp. 6), 1-3, 2006.

[3] McNamara J. O., Pharmacotherapy of the epilepsies. Brunton L.L., Lazo J. S., Parker K. L., Goodman \& Gilman's the pharmacological basis of therapeutics. 11th ed. New York. McGraw hill. 2006, 521-548.

[4] Lowenstein D. H., Seizures and epilepsy. Kasper D. L., Braunwald E., Fauci A. S., Hauser S. L., Longo D. L., Jameson J. L., Harrison's principles of internal medicine. $17^{\text {th }}$ ed. New York. McGraw hill. 2008, 2357-2371.

[5] Available: http://en.wikipedia.org/wiki/GABAA_receptor. [Accessed Nov. 29, 2013]. 
[6] Amir M., Hasan M. Z., "Functional roles of benzothiazole motif in antiepileptic drug research", Mini reviews in medicinal chemistry, Nov. 2013. (PubMed ID 24251804).

[7] Nadeem S., et. al., "Synthesis and preliminary screening of benzothiazol-2-yl thiadiazole derivatives for anticonvulsant activity”, Acta Pharm., 59, 441-451, 2009.

[8] Harish R., et. al., "Synthesis and antiepileptic activity of some novel semicarbazones containing 1, 3, 4-thiadiazole and quinazoline ring”, Acta Poloniae Pharmaceutica-Drug Research, 69(2), 253-261, 2012.

[9] Siva Kumar R., Nafeez Basha S. K., Kumarnallasivan P., Vijaianand P. R., Pradeepchandran R., "A computational design and docking studies on Escherichia coli b-Ketoacyl-Acyl carrier protein synthese III using auto dock", J Pharm Res, 3(7), 14601462, 2010.
[10] Ajeet, "In silico designing and characterization of Amiloride derivatives as ion channel modulator”, Med Chem Res, 22(2), 1004-1010, 2013.

[11] Ajeet, "Trans-disciplinary receptor binding of acyclovir to human phenylalanine hydroxylase: docking approach”, International Journal of Pharmacy and Pharmaceutical Sciences, 4(suppl 2), 182-184, 2012.

[12] Ajeet, Tripathi L., Kumar P., "Designing of Novel 6(H)-1, 3, 4Thiadiazine Derivatives as MMP12 Inhibitors: A MLR and Docking Approach", American Journal of Pharmacological Sciences, 1 (2), 29-34, 2013.

[13] Mills N., "ChemDraw Ultra 10.0.", J. Am. Chem. Soc., 128(41), 13649-13650, 2006.

[14] Yap, C. W., "PaDEL-descriptor: An open source software to calculate molecular descriptors and fingerprints," J. Comput. Chem. 32(7), 1466-1474, 2011. 